INFLAMMATORY BOWEL DISEASE

\title{
Infliximab in moderately severe glucocorticoid resistant ulcerative colitis: a randomised controlled trial
}

\author{
C S J Probert, S D Hearing, S Schreiber, T Kühbacher, S Ghosh, I D R Arnott, A Forbes
}

Gut 2003;52:998-1002

See end of article for authors' affiliations

Correspondence to: Dr C S J Probert, University Division of Medicine, Bristol Royal Infirmary, Marlborough St, Bristo BS2 8HW, UK;

c.s.j.probert@bristol.ac.uk

Accepted for publication 21 March 2003
Background: Tumour necrosis factor production is increased in the mucosa of patients with active ulcerative colitis. The benefits of infliximab in Crohn's disease are established. We investigated its efficacy in ulcerative colitis.

Methods: We conducted a randomised placebo controlled trial of infliximab $(5 \mathrm{mg} / \mathrm{kg})$ in the treatment of glucocorticoid resistant ulcerative colitis. Infusions were given at weeks 0 and 2 . Disease activity and quality of life were recorded over eight weeks of follow up. Remission was defined as an ulcerative colitis symptom score (UCSS) of $\leqslant 2$ and/or Baron score of 0 at week 6 . Patients not in remission were offered open label infliximab $10 \mathrm{mg} / \mathrm{kg}$ and reviewed two weeks later.

Results: After two weeks, there was no statistically significant difference between the infliximab and placebo groups in the proportion of patients with a Baron score of $0(13 \%(3 / 23) \vee 5 \%(1 / 19)(95 \%$ confidence interval $(\mathrm{Cl})-9 \%$ to $24 \%) ; p=0.74$ ). After six weeks, remission (UCSS $\leqslant 2$ ) rates were $39 \%$ $(9 / 23)$ versus $30 \%(6 / 20)(95 \% \mathrm{Cl}-19$ to $34 \% ; p=0.76)$. The median improvement in UCSS was 3 for the infliximab group and 2.5 for the placebo group ( $p=0.82$, Mann-Whitney $U$ test). A Baron score of 0 was likely in either group $(26 \%(6 / 23)$ v $30 \%(6 / 20)(95 \% \mathrm{Cl}-30 \%$ to $23 \%) ; p=0.96)$. Improvement in the IBDQ and EuroQol was not significantly different between the groups $(p=0.22$ and 0.3 , respectively, Mann-Whitney $U$ test). Twenty eligible patients were given open labelled infusions. Remission was achieved in $3 / 11$ (27\%) patients initially treated with infliximab and in 1/9 (11\%) patients treated with placebo.

Conclusion: These data do not support the use of infliximab in the management of moderately active glucocorticoid resistant ulcerative colitis.

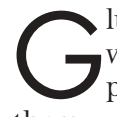
ucocorticoid resistance affects up to $30 \%$ of patients with severe ulcerative colitis. ${ }^{1-3}$ Although it may be possible to predict failure to respond to glucocorticoid therapy in some patients, ${ }^{12}$ little can be done to alter glucocorticoid resistance at present. Some of these patients may respond to cyclosporin ${ }^{34}$ but are likely to undergo colectomy within the next $6-12$ months. ${ }^{56}$ The role of increased production of tumour necrosis factor $\alpha$ (TNF) has been established in the pathophysiology of both ulcerative colitis and Crohn's disease: in 1991, Murch et al reported that, during relapse, serum concentrations of TNF in patients with inflammatory bowel disease were significantly greater than those in healthy controls. ${ }^{7}$ Median serum concentration of TNF in ulcerative colitis $(27 \mathrm{pg} / \mathrm{ml})$ was substantially greater than that in Crohn's disease ( $16 \mathrm{pg} / \mathrm{ml})$. Increased concentrations of TNF have also been reported in the mucosa of patients with active ulcerative colitis. ${ }^{8-10}$

Increased levels of TNF production have given rise to the development of therapies to neutralise this cytokine. The benefits of several monoclonal antibodies against TNF have been established in Crohn's disease. ${ }^{11-14}$ Infliximab, a chimeric antiTNF monoclonal antibody, is a potent anti-inflammatory agent that effectively blocks the action of TNF. Onset of the clinical effect is usually seen within days. ${ }^{11}{ }^{15}$ The mechanism of action may include neutralisation of TNF, lysis of activated immune cells, ${ }^{16}$ and induction of apoptosis in activated macrophages ${ }^{17}$ and T cells. ${ }^{18}$ Application of an antibody to TNF was effective in an animal model of colitis. ${ }^{19}$ Watkins et al reported a similar benefit in cottontop tamarins that can develop a disease resembling human ulcerative colitis spontaneously. ${ }^{20}$

There are very few clinical data on the role of antibodies to TNF in the therapy of ulcerative colitis. ${ }^{21}$ During the course of our study, several reports have appeared. Most were open studies that suggested some benefit from infliximab. However, one was a controlled trial that was terminated prematurely due to poor recruitment..$^{22}$ There are also several case reports with a range of outcomes.

We conducted a double blind, randomised, placebo controlled trial of infliximab in the treatment of moderately severe glucocorticoid resistant ulcerative colitis.

\section{METHODS}

Study design

In a randomised, double blind, placebo controlled study conducted in four centres in the UK and Germany, we evaluated the role of infliximab in the treatment of patients with moderately severe glucocorticoid resistant ulcerative colitis. The study protocol was approved by the research ethics committee for each centre and all patients gave written informed consent prior to recruitment.

\section{Patients}

The trial involved male and female patients aged 18 years or more. Patients had (i) an established diagnosis of ulcerative colitis, (ii) failed to respond to conventional treatment with glucocorticoids, and (iii) were not in need of urgent colectomy. Diagnosis and extent of ulcerative colitis were established prior to the present active phase for which the patient was being treated when recruited. Patients had to have received conventional treatment with at least $30 \mathrm{mg}$ prednisolone (or equivalent) for at least one week, for relapse, but still had

Abbreviations: TNF, tumour necrosis factor $\alpha$ : UCSS, ulcerative colitis symptom score; $Q O L$, quality of life; IBDQ, QOL index for inflammatory bowel disease; $M W$, Mann-Whitney $U$ test; $C R P, C$ reactive protein. 
clinical activity that qualified for inclusion in the study. At screening, all patients had to have an ulcerative colitis symptom score (UCSS) $)^{23}$ of 6 or more and a sigmoidoscopy score of at least 2 on the Baron scale. ${ }^{24}$ In addition, all patients had a biopsy taken showing histological changes of acute ulcerative colitis.

Patients were excluded from the trial if they had fulminant disease likely to require colectomy. We specifically excluded patients with severe disease rather than give them either an unlicensed drug or placebo. Women who were pregnant or were planning a pregnancy during or within six months of the trial were excluded. Patients who had received cyclosporin, any therapeutic agent used to directly reduce TNF, or any investigational drug within three months of enrolment, as well as those who had recently commenced treatment (within the last three months) with 6-mercaptopurine or azathioprine were also excluded. However, patients who were receiving a stable dose of 6-mercaptopurine or azathioprine for more than three months were not excluded. All patients underwent stool examination; any patient with a gastrointestinal infection was excluded. Those with known serious infections (such as hepatitis, pneumonia, pyelonephritis, or an opportunistic infection) in the previous three months were excluded. Patients with past or current colorectal dysplasia in the opinion of the local pathologist, malignancy, or Crohn's disease were excluded. Finally, prior intestinal resection, other than appendicectomy, or the presence of a stoma were also exclusion criteria.

\section{Recruitment and randomisation}

Consecutive patients who met the inclusion and exclusion criteria were recruited. Each centre was allocated a series of patient numbers by Schering-Plough. Each number was randomly assigned to either treatment group based on blocks of four within each centre. The local pharmacists and investigators were blinded to the treatment each patient received

\section{Treatment}

All patients were randomised to receive a blinded infusion of infliximab $5 \mathrm{mg} / \mathrm{kg}$ body weight or placebo at week 0 and a second identical infusion at week 2 . At week 6 , all patients were reassessed and those who continued to have active ulcerative colitis were offered open label treatment with $10 \mathrm{mg} / \mathrm{kg}$ infliximab. The second infusion was given to see whether it offered any additional benefit over the first. The option to receive open label treatment was included to ensure non-responders who may have received placebo had the opportunity to have active treatment, in addition to determining whether the third dose could bring about remission in those patients who failed to respond to the first two doses. Importantly, at that stage, the investigators remained blinded to the nature of the earlier infusions.

Infliximab was administered according to the manufacturer's instructions. The placebo was identical in appearance and contained $2.2 \mathrm{mg}$ monobasic sodium phosphate monohydrate, $6.1 \mathrm{mg}$ dibasic sodium phosphate dihydrate, $500 \mathrm{mg}$ sucrose, and $0.5 \mathrm{mg}$ polysorbate-80. Infusions were delivered over a two hour period. After infusion patients were observed for a further two hours.

Antirheumatic drugs, antibiotics, and antidiarrhoeal drugs were forbidden during the study. Paracetamol (acetaminophen) was used if analgesic medication was required. The dose of 5-aminosalicylic acid was kept stable throughout the study. The dose of glucocorticoids was kept stable during screening but was then allowed to be changed according to clinical demands. The aim was to reduce the daily dose of glucocorticoid by $5 \mathrm{mg}$ of prednisolone equivalent each week. Azathioprine/6-mercaptopurine doses were kept constant.

\section{Outcome measures}

Disease activity was assessed at recruitment and six weeks after the first infusion using the UCSS. ${ }^{23}$ The UCSS is calculated from four observations: stool frequency, rectal bleeding, sigmoidoscopic appearance, and the physician's global assessment. Sigmoidoscopic assessment of disease activity was performed at recruitment, two, six, and eight weeks after the first infusion, using the Baron scale. ${ }^{24}$ The same endoscopist, who was blinded to the treatment, was used at each of the centres throughout the study. Two hard end points were utilised to indicate remission: clinical remission defined as UCSS $\leqslant 2$ and sigmoidoscopic remission as a Baron score of 0 .

Secondary end points were changes in UCSS, Baron score, quality of life (QOL), and serum $C$ reactive protein (CRP) levels, and change in daily glucocorticoid dose. QOL was measured using the QOL index for inflammatory bowel disease (IBDQ) ${ }^{25}$ and EuroQol ${ }^{26}$ at the start of the study and six weeks after the first infusion.

The outcome of patients in each arm receiving open label treatment with $10 \mathrm{mg} / \mathrm{kg}$ infliximab at week 6 was also recorded. Serious adverse events during the study period and the following 30 days were recorded in compliance with good clinical practice. Serious adverse events were defined according to the latest International Committee on Harmonisation. This included patients who underwent colectomy during the study period and the following 30 days.

\section{Statistical analysis}

Descriptive statistics are presented. Standard deviations are shown when means have been calculated or compared. Interquartile ranges are shown with medians. $\chi^{2}$ tests were performed with Yates' continuity correction for $2 \times 2$ tables, or without correction for larger tables; $95 \%$ confidence intervals (CI) are shown for differences in proportions. The MannWhitney U test (MW) was performed using SPSS software by an independent statistician. No centre effects were seen.

Sample size calculations were based on assumptions from published trails of infliximab in Crohn's disease. Targan and colleagues ${ }^{11}$ reported an $81 \%$ response rate to treatment with infliximab $5 \mathrm{mg} / \mathrm{kg}$ for Crohn's disease. A placebo response rate of $30 \%$ may be expected for patients with ulcerative colitis. ${ }^{27}$ Sample size calculations showed that 44 patients were needed to show a difference of $51 \%$ with $95 \%$ confidence and $90 \%$ power.

\section{RESULTS}

\section{Patient population}

Forty three patients were randomised: 13 from Bristol, 15 from St Mark's Hospital, eight from Kiel, and seven from Edinburgh. Population demographics and disease characteristics are summarised in table 1 . These characteristics were similar in both groups. All had received at least $30 \mathrm{mg}$ prednisolone (or equivalent) daily for one week or longer in the course of their current exacerbation. In fact, 33/43 (77\%) had received glucocorticoid treatment for 14 days or more and median duration of such treatment was 28 days in both groups. Such was the concern about the duration of glucocorticoid treatment that although 33 patients continued to receive $30 \mathrm{mg}$ or more at the time of inclusion, in 10 cases failure to achieve satisfactory remission from glucocorticoid therapy had led to commencement of weaning from glucocorticoids to minimise drug induced morbidity.

\section{Clinical end points}

After six weeks, there was no statistically significant difference between the rate of remission (UCSS $\leqslant 2$ ) in the infliximab and placebo groups (39\% (9/23) v 30\% (6/20) $(95 \%$ CI for difference -19 to $34 \%$ ); $\chi^{2}=0.09 ; \mathrm{p}=0.76$ ) (table 2 ). One patient from the placebo group underwent colectomy during the intervention period and was recorded as a treatment failure. The mean improvement in UCSS was 4 (SD 3) for both groups (table 2). The median improvement in UCSS was 3 for 
Table 1 Demographic and disease characteristics of the patients at week 0

\begin{tabular}{lll} 
& Placebo group & Infliximab group \\
\hline Age & $40(29-43.5)$ & $41(35.5-50.5)$ \\
Weight (kg) & $72(60-8)$ & $66(61-78)$ \\
Extensive UC & 13 & 14 \\
Left sided disease & 3 & 5 \\
Distal colitis & 4 & $45(39-141)$ \\
Duration of UC (months) & $59(35-96)$ & 26 \\
Azathioprine use (\%) & 35 & $30(30-30)$ \\
Prednisolone equivalent (mg/day) & $30(25-30)$ & $28(11.5-42)$ \\
Duration of steroid treatment (days) & $28(14-45)$ & \\
\hline Data are shown as medians (interquartile ranges). Glucocorticoid doses have been converted to prednisolone \\
equivalents for those patients receiving 6-methyl prednisolone. \\
UC, ulcerative colitis.
\end{tabular}

Table 2 Effect of treatment with infliximab or placebo

\begin{tabular}{|c|c|c|c|c|c|c|}
\hline \multirow[b]{2}{*}{ Group } & \multicolumn{2}{|l|}{ Week 0} & \multicolumn{2}{|l|}{ Week 6} & \multicolumn{2}{|c|}{ Improvement } \\
\hline & $\begin{array}{l}\text { Placebo } \\
(n=20)\end{array}$ & $\begin{array}{l}\text { Infliximab } \\
(n=23)\end{array}$ & Placebo & Infliximab & Placebo & Infliximab \\
\hline Prednisolone equivalent (mg/day) & 28 (7) & $32(11)$ & $14(10)$ & $13(12)$ & $14(12)$ & $19(15)$ \\
\hline $\mathrm{C}$ reactive protein & 12 (10) & $9(9)$ & $13(14)$ & $9(8)$ & $-1(10)$ & $0(10)$ \\
\hline UC severity score & $8.5(2)$ & $8(2)$ & $5(3)$ & $5(3)$ & $4(3)$ & $4(3)$ \\
\hline Baron score & $2.4(0.5)$ & $2(0.5)$ & $1(1)$ & $1(1)$ & $1(1)$ & $1(1)$ \\
\hline $\mathrm{IBDQ}$ & 114 (29) & $127(40)$ & $139(43)$ & $163(40)$ & $25(28)$ & $36(49)$ \\
\hline EuroQol & 49 (17) & $52(16)$ & $54(23)$ & $59(19)$ & $4(16)$ & $7(17)$ \\
\hline
\end{tabular}

Data are shown as means (standard deviation). Glucocorticoid doses have been converted to prednisolone equivalents for those patients receiving 6- methyl prednisolone.

UC, ulcerative colitis; $Q O L$, quality of life; IBDQ, $Q O L$ index for inflammatory bowel disease.

the infliximab group and 2.5 for the 18 assessable members of the placebo group. This difference was not statistically significant ( $M W, p=0.82)$.

\section{Sigmoidoscopic end points}

After two weeks, there was no statistically significant difference between the infliximab and placebo groups in the proportion of patients with a Baron score of $0(13 \%(3 / 23) v 5 \%$ $(1 / 19)(95 \%$ CI for difference $-9 \%$ to $\left.24 \%) ; \chi^{2}=0.10 ; p=0.74\right)$. In $17(74 \%)$ patients in the infliximab group and $12(63 \%)$ in the placebo group, the Baron score did improve, as defined by a decrease in score of at least 1 (95\% CI for difference -14 to $42 ; \chi^{2}=0.17 ; \mathrm{p}=0.68$ ).

At week 6 , there was no statistically significant difference between the infliximab and placebo groups in the proportion of patients with a Baron score of $0(26 \%(6 / 23) v 30 \%(6 / 20)$ ( $95 \%$ CI for difference $-30 \%$ to $23 \%$ ); $\chi^{2}=0.003 ; p=0.96$ ) (table 2). The mean improvement in Baron score was 1 in each group (SD 1 for each group). Thirteen (57\%) patients in the infliximab group improved, seven $(30 \%)$ remained the same, and three $(13 \%)$ deteriorated such that the score increased by at least 1 point; 11 (58\%) patients in the placebo group improved, seven $(37 \%)$ remained the same, and one underwent colectomy $\left(\chi^{2}\right.$ without Yates' continuity correction $=0.79$; $\mathrm{p}=0.67$ ). One patient refused sigmoidoscopic assessment but by other clinical measures was deemed to be a treatment failure.

\section{$\mathrm{C}$ reactive protein}

From week 0 to week 6, median levels of CRP rose in patients who received infliximab, from 6.5 to $10 \mathrm{mg} / \mathrm{l}$, but the median value did not change in the placebo group. When changes in CRP were compared, no difference was found ( $M W, p=0.96)$.

\section{Quality of life}

Mean IBDQ rose by 36 in the infliximab group (SD 49) compared with 25 in the placebo group (SD 28). The results suggest a modest benefit from infliximab (MW, $\mathrm{p}=0.22)$ (table 2 ).
Mean improvement in EuroQol was 7 in patients who received infliximab (SD 17) and 4 in whose who received placebo (SD 16), (MW, p=0.3).

\section{Glucocorticoid use}

Most patients in each group were able to reduce their dose of glucocorticoid. Mean reduction in daily dose of glucocorticoid in the infliximab group was equivalent to $19 \mathrm{mg}$ prednisolone (SD 15) compared with $14 \mathrm{mg}$ (SD 12) in the placebo group (table 2). This difference was just statistically significant (MW, $\mathrm{p}=0.037)$.

Effect of disease extent and immunosuppressive agents The groups were well matched for disease duration and extent. Extent of disease had no bearing on remission rate. Of those patients who received infliximab, 5/14 (36\%) with total colitis went into remission, $3 / 5(60 \%)$ with left sided colitis and $1 / 4(25 \%)$ with distal colitis ( $\chi^{2}$ without Yates' continuity correction $=1.3 ; p=0.5)$. When remission rates of patients with total disease in each of the two groups were compared, no significant difference was found $\left(\chi^{2}\right.$ without Yates' continuity correction $=0.02 ; \mathrm{p}=0.9$ ).

The difference in remission rate among the 12 patients who were receiving azathioprine did not reach statistical significance $(2 / 6(33 \%)$ receiving placebo $v 4 / 6(67 \%)$ receiving infliximab ( $95 \%$ CI for difference $-79 \%$ to $45 \%)$; $\chi^{2}=0.02$; $\mathrm{p}=0.89$ ) although the study was under powered to adequately address this issue.

\section{Open label infusions}

Open labelled infusion of infliximab $10 \mathrm{mg} / \mathrm{kg}$ body weight was given to 20 eligible patients with continued active disease at week 6. Clinical remission was achieved in 3/11 (37\%) patients who had received prior treatment with infliximab at week 0 and week 2 and 1/9 (11\%) patients previously treated with placebo. Thus $1 / 9(11 \%)$ patients who received treatment with infliximab for the first time went into remission. The 
median improvement in the Baron score was 1. CRP fell in four patients and rose in one. The median change in CRP was 0 .

\section{Serious adverse events}

Two serious adverse events, which qualified as life threatening or severe, were recorded. Both affected the placebo group. One patient suffered septic complications. Another underwent colectomy because of toxic exacerbation and spontaneous perforation. All other serious adverse events were rated as mild and were not significantly different in frequency between infliximab and placebo treated patients. No significant infusion reactions were seen.

\section{DISCUSSION}

Twenty three patients received infliximab for moderately severe glucocorticoid resistant ulcerative colitis. By week 2, sigmoidoscopic remission was apparent in three patients who had received infliximab compared with one who had received placebo, and by week 6 this value had increased to 6 for both patient groups. By most outcome measures, there was no statistically significant difference between the infliximab and placebo groups. While the IBDQ improved slightly with infliximab, this was not statistically significant. However, there was a significantly greater reduction in the dose of glucocorticoid in patients who received infliximab but this is difficult to interpret as the tapering schedule was not standardised and was dependent on the physician's assessment. Infliximab was well tolerated and there were no serious adverse events among those patients who received active treatment.

An indicator of the poor outcome of the trial was that 20 patients elected to have open labelled treatments for continued active disease. An infusion of $10 \mathrm{mg} / \mathrm{kg}$ body weight infliximab did not result in a substantial increase in the frequency of endoscopic remission.

There were no adverse reactions to infusion, which for 11 patients was their third in six weeks. Ongoing glucocorticoid therapy and the high frequency of infliximab infusions may have protected patients from potential infusion reactions mediated by antibodies to infliximab, which have been described in other clinical trials. The main adverse reactions, sepsis and colectomy because of worsening disease, both occurred in the placebo group.

In the first report of anti-TNF antibody to treat ulcerative colitis, 15 patients received open label treatment with CDP571. ${ }^{21}$ A significant reduction in the Powell-Tuck score was noted one week after infusion but this benefit was not sustained. The improvement in sigmoidoscopic score and in the number of liquid stools was not statistically significant Chey and colleagues ${ }^{28}$ reported a series of eight patients treated with a single dose of infliximab. All apparently responded extremely well, entering and remaining in remission for up to five months. Both studies used open label treatment and appeared to show some benefit to patients. Some patients who received study drug in our study also improved. However, the rate of improvement of patients in the active treatment arm was no better than that in the placebo arm. This emphasises the crucial role of randomised placebo controlled trials in the evaluation of new drugs.

In the trial reported by Sands and colleagues, ${ }^{22}$ eight patients were randomised to receive infliximab (three receiving $5 \mathrm{mg} / \mathrm{kg}$, three $10 \mathrm{mg} / \mathrm{kg}$, and two $20 \mathrm{mg} / \mathrm{kg}$ ), and three patients received placebo: $50 \%$ of infliximab treated patients were reported to have responded by two weeks. Failure to respond was defined as failure to achieve a clinical response based on the Truelove and Witts assessment score, if the patient had to receive an increased dose of glucocorticoid or immunomodulator because of a lack of clinical response, or if the patient underwent colectomy or died. Of the treatment failures, one underwent colectomy during the first two weeks and three had a colectomy during the follow up period. In a tacit acknowledgement that the study was under powered, no statistical analysis was offered by the authors.

Some open studies have suggested the infliximab may be efficacious. ${ }^{28-30}$ Two studies of glucocorticoid resistant ulcerative colitis in similar populations came to opposite conclusions: Kohn and colleagues ${ }^{30}$ reported that $10 / 13$ (77\%) patients had a clinical response to a single infusion of infliximab, nine of whom $(70 \%$ of the total) had a sustained response. Conversely, Actis and colleagues ${ }^{31}$ found that $4 / 8$ patients $(50 \%)$ responded initially and only two had a sustained response. More recently, Su and colleagues ${ }^{32}$ reported open treatment in 27 patients; remission was achieved in $12(44 \%)$. However, they observed that remission or partial response was only achieved in $3 / 9$ (33\%) of what they termed "steroid refractory" patients. Two patients in their series developed serious adverse events, one of whom died.

We have found no statistically significant benefit to patients from adding infliximab to standard therapy. It is possible that our definition of glucocorticoid resistance was not strict enough and that waiting longer may have selected out a more resistant group of subjects but the median period for which patients had received glucocorticoid therapy prior to recruitment was 28 days. There is no internationally accepted definition of glucocorticoid resistance; some investigators have used failure at 14 days rather than seven. In our study, $77 \%$ of patients would have met that stricter definition. Furthermore, the rate of complete remission at week 6 was poor in both the infliximab and placebo arms of the study (39\% and 30\%, respectively, using the UCSS, or $26 \%$ and $30 \%$ using sigmoidoscopy scoring alone) suggesting that patients were resistant to glucocorticoid therapy and the definition was reasonable. We would expect that a less restrictive definition of glucocorticoid resistance may be associated with a higher response rate to immunosuppression (including infliximab) than a more restricted one. The placebo response in the trial presented here is not at odds with the rates of $16-52 \%$ cited by Hanauer. ${ }^{27}$ This is interesting when compared with the $33 \%$ response rate to infliximab among "steroid refractory" patients reported by $\mathrm{Su}$ and colleagues. ${ }^{32}$

Our end point of remission, based on the endoscopic score or UCSS, was stricter than the efficacy measure used by Sands and colleagues. ${ }^{22}$ We would argue that any new treatment must make an impact on the remission rate if it is to be used in this setting. Simply prolonging glucocorticoid therapy with or without other therapeutic agents exposes patients to potentially harmful side effects. For patients in whom remission is not achieved in a reasonable period of time, colectomy should be considered.

Forty three patients were randomised (20:23) in our study, resulting in $90 \%$ power to find a $51 \%$ difference $(30 \%$ placebo to $81 \% 5 \mathrm{mg} / \mathrm{kg}$ infliximab) with $95 \%$ confidence. Thus it was not powered to find a smaller difference, raising the possibility of a type II error, although a lower response rate would have to be balanced against the potential side effects of therapy with infliximab. ${ }^{32}{ }^{33}$ Importantly, if the lack of statistical significance were simply due to a lack of power, then a numeric difference in remission rates or any of the secondary end points studied would be evident. However, the summary data for each group at each time point (CRP, response rates, QOL) remained remarkably similar with no trend in favour of infliximab evident.

It might be argued that some benefit was evident at week 2 . When the proportions of patients from each group in endoscopic remission were compared, confidence intervals were wide and suggested a benefit of up to $24 \%$ in favour of infliximab. By week 6 , the upper confidence limit for the benefit from infliximab was $34 \%$ when assessed by UCSS $\leqslant 2$. However, the benefit was reversed when remission at week 6 was assessed by endoscopy alone: the upper confidence limit for the benefit was $23 \%$, associated with placebo. Hence while 
the post hoc power calculation indicated that the study should be able to detect a $51 \%$ difference, mirroring the improvement found in patients with Crohn's disease, the study may have been inadequately powered to be sure that there is no clinically relevant benefit from infliximab.

Our data do not support the widespread use of infliximab in the management of glucocorticoid resistant ulcerative colitis. The small advantage of infliximab in glucocorticoid sparing deserves further exploration in controlled trials. It is possible that, with refinement of laboratory techniques, a subgroup of responders may be found, but clinical observation alone has not shown any role for this drug in unselected glucocorticoid resistant patients with ulcerative colitis.

\section{ACKNOWLEDGEMENTS}

We are grateful to Jochen Grebe, Meron Jacyna, Dick Mountford, Susanna Nikolaus, Max Pitcher, and Ajeya Shetty for their contributions to case selection and clinical care. Institutional grant support was received from Schering-Plough and from the BMBF Competence Network "Chronic Inflammatory Bowel Disease" (Germany). We are indebted to Hazel Taylor for performing the statistical analysis. We wish to thank Schering Plough Ltd (UK) and Essex Pharma (Germany) for providing the drugs used in this Investigator Instigated Study.

\section{Authors' affiliations}

C S J Probert, S D Hearing, University Division of Medicine, Bristol Royal Infirmary, Bristol, UK,

S Schreiber, T Kühbacher, First Medical Department

Christian-Albrechts-University, Kiel, Germany

S Ghosh, I D R Arnott, Western General Hospital, Edinburgh, UK

A Forbes, St Mark's Hospital, Harrow, London, UK

\section{REFERENCES}

1 Travis SPL, Farrant JM, Nolan DJ, et al. Predicting outcome in severe ulcerative colitis. Gut 1996;38:905-10.

2 Hearing SD, Norman M, Probert CSJ, et al. Predicting therapeutic outcome in severe ulcerative colitis by measuring in vitro steroid sensitivity of proliferating peripheral blood lymphocyte. Gut 1999;45:382-8.

3 Sandborn WJ. Cyclosporine in ulcerative colitis: state of the art. Acta Gastroenterol Belg 2001;64:201-4.

4 Lichtiger S, Present DH, Kornbluth A, et al. Cyclosporine in severe ulcerative colitis refractory to steroid therapy. N Engl J Med 1994;330: 1841-5.

5 Hyde GM, Thillainayagam AV, Jewell DP. Intravenous cyclosporin as rescue therapy in severe ulcerative colitis: time for a reappraisal? Eur Gastroenterol Hepatol 1998:10:411-13.

6 Haslam N, Hearing SD, Probert CSJ. Audit of cyclosporin use in inflammatory bowel disease: limited benefits, numerous side-effects. Eur J Gastroenterol Hepatol 2000; 12:657-60

7 Murch SH, Lamkin VA, Savage MO, et al. Serum concentrations of tumour necrosis factor alpha in childhood chronic inflammatory bowel disease Gut 1991:32:913-17.

8 MacDonald TT, Hutchings P, Choy MY, et al. Tumour necrosis factor-alpha and interferon-gamma production measured at the single cell level in normal and inflamed human intestine. Clin Exp Immunol 1990;81:301-5.
9 Murch SH, Braegger CP, Walker-Smith JA, MacDonald TT. Location of tumour necrosis factor alpha by immunohistochemistry in chronic inflammatory bowel disease. Gut 1993:34:1705-9.

10 Reinecker HC, Steffen M, Witthoett T, et al. Enhanced secretion of tumor-necrosis- factor alpha, IL-6, and IL-1 -BETA by isolated lamina propria mononuclear-cells from patients with ulcerative colitis and Crohn's disease. Clin Exp Immunol 1993;94:174-81.

11 Targan SR, Hanauer SB, van Deventer Sj, et al. A short-term study of chimeric monoclonal antibody cA2 to tumor necrosis factor alpha for Crohn's disease. N Engl J Med 1997;337:1029-35.

12 Present DH, Rutgeerts P, Targan S, et al. Infliximab for the treatment of fistulas in patients with Crohn's disease. N Engl J Med 1999;349:1398-405.

13 Stack WA, Mann SD, Roy AJ, et al. Randomised controlled trial of CDP571 antibody to tumour necrosis factor-alpha in Crohn's disease. Lancet 1997;349:521-4

14 Arnott IDR, McDonald D, Williams A, Ghosh S. Clinical use of Infliximab in Crohn's disease: the Edinburgh experience. Aliment Pharmacol Ther 2001;15:1639-46.

15 Nikolaus S, Raedler A, Kuhbacher T, et al. Mechanisms in failure of infliximab for Crohn's disease. Lancet 2000;356:1475-9.

16 Scallon BJ, Moore MA, Trinth H, et al. Chimeric anti-TNF-alpha monoclonal- antibody CA2 binds recombinant transmembrane TNF-alpha and activates immune effector functions. Cytokine 1995; 7:251-9

17 Schmidt $M$, Luegering $N$, Luegering $A$, et al. Infliximab exerts anti-inflammatory capacity in IBD by induction of apoptosis in monocytes. Gastroenterology 2000;118(suppl 2):3630.

18 ten Hove T, van Monffrans C, Peppelenbosch MP, van Deventer SJH. Infliximab treatment induces apoptosis of lamina propria T lymphocytes in Crohn's disease. Gut 2002:50:206-11.

19 Ward PS, Woodger SR, Bodmer M, Foulkes R. Anti-tumour necrosis factor alpha monoclonal antibodies are therapeutically effective in a model of colonic inflammation. Br J Pharmacol 1993; 1 10:77P.

20 Watkins PE, Warren BF, Stephens S, et al. Treatment of ulcerative colitis in the cottontop tamarin using antibody to tumour necrosis factor alpha. Gut 1997; 40:628-33

21 Evans RC Clarke L, Heath $P$, et al. Treatment of ulcerative colitis with an engineered human anti-TNF alpha antibody CDP571. Aliment Pharmacol Ther 1997:11:1031-5.

22 Sands BE, Tremaine WJ, Sandborn WJ, et al. Infliximab in the treatment of severe, steroid-refractory ulcerative colitis: a pilot study. Inflamm Bowel Dis $2001 ; 7: 83-8$.

23 Schroeder KW, Tremaine WJ, Ilstrup DM. Coated oral 5-aminosalicylic acid therapy for mildly to moderately active ulcerative colitis-a randomized study. N Engl J Med 1987;317:1625-9.

24 Baron JH, Connell AM, Lennard-Jones JE. Variation between observers in describing mucosal appearances in proctocolitis. BMV 1964;i:89-92

25 Irvine EJ. A quality-of-life index for inflammatory bowel disease. Can J Gastroenterol 1993;7:155-9.

26 Williams A for EuroQol Group. EuroQol-a new facility for the measurement of health-related quality of life. Health Policy 1990;16:199-208

27 Hanauer SB. Medical therapy for ulcerative colitis. In: Kirsner JB, ed. Inflammatory Bowel Disease, 5th edn. Philadelphia: W B Saunders, 2000.

28 Chey WY, Hussain A, Ryan C, et al. Infliximab for refractory ulcerative colitis. Am J Gastroenterol 2001:96:2373-81.

29 Kaser A, Marringer T, Voel T, et al. Infliximab in severe steroid-refractory ulcerative colitis: A pilot study. Wien Klin Wochenschr $2001 ; 113: 930-33$

30 Kohn A, Prantera C, Pera A, et al. Anti-tumour necrosis factor (Infliximab) in the treatment of severe ulcerative colitis: result of an open study on 13 patients. Dig Liver Dis 2002;34:626-30.

31 Actis GC, Bruno $M$, Pinna-Pintor $M$, et al. Infliximab for treatment of steroid- refractory ulcerative colitis. Dig Liver Dis 2002;34:631-4.

32 Su C, Salzberg BA, Lewis JD, et al. Efficacy of anti-tumor necrosis factor therapy in patients with ulcerative colitis. Am J Gastroenterol therapy in patients with

33 Keane J, Gershon S, Wise RP, et al. Tuberculosis associated with infliximab, a tumor necrosis factor $\alpha$-neutralizing agent. N Engl J Med 2001:345:1098-104. 